\title{
La Lettre et l'œuvre. Correspondances de Marguerite Yourcenar, textes réunis par André-Alain Morello
}

\section{Susanna Bonomi}

\section{(2) OpenEdition}

1 Journals

\section{Edizione digitale}

URL: http://journals.openedition.org/studifrancesi/6093

DOI: $10.4000 /$ studifrancesi.6093

ISSN: 2421-5856

\section{Editore}

Rosenberg \& Sellier

\section{Edizione cartacea}

Data di pubblicazione: 1 mai 2011

Paginazione: 212

ISSN: 0039-2944

\section{Notizia bibliografica digitale}

Susanna Bonomi, «La Lettre et l'œuvre. Correspondances de Marguerite Yourcenar, textes réunis par André-Alain Morello», Studi Francesi [Online], 163 (LV | I) | 2011, online dal 30 novembre 2015, consultato il 13 janvier 2021. URL: http://journals.openedition.org/studifrancesi/6093 ; DOI: https:// doi.org/10.4000/studifrancesi.6093

Questo documento è stato generato automaticamente il 13 janvier 2021.

\section{(c)}

Studi Francesi è distribuita con Licenza Creative Commons Attribuzione - Non commerciale - Non opere derivate 4.0 Internazionale. 


\title{
La Lettre et l'œuvre. Correspondances de Marguerite Yourcenar, textes réunis par André-Alain Morello
}

\author{
Susanna Bonomi
}

\section{NOTIZIA}

La Lettre et l'œuvre. Correspondances de Marguerite Yourcenar, textes réunis par

André Alain MORELLO, Paris, Honoré Champion, 2009 («Babeliana»), pp. 297.

1 Questo volume, come scrive il suo curatore André-Alain MORELLo, è «le prolongement» del Colloquio internazionale tenutosi il 9 e 10 dicembre 2004 presso l'Université du Sud Toulon-Var. Gli esperti, intervenuti al dibattito, sono stati chiamati a riflettere sul vasto corpus di lettere conservato maniacalmente da Marguerite Yourcenar e, in parte, già pubblicato: Lettres à ses amis et quelques autres (1995), D'Hadrien à Zénon. Correspondance 1951-1956 (2004) e «Une volonté sans fléchissement». Correspondance 1957-1960 (2007).

2 Il titolo di questa raccolta di atti, La Lettre et l'œuvre, indica subito uno dei punti focali della discussione: la corrispondenza della Yourcenar non è solo strettamente collegata alla sua opera, ma è parte della stessa. Proprio da questa considerazione prende le mosse Jean-Pierre CASTELLANI (La Correspondance de Marguerite Yourcenar: discours à soimême ou discours aux autres, pp. 21-32) che, esaminando la corrispondenza reale e fittizia (le lettere inserite nelle opere) dell'autrice, conclude che, grazie alla lettera "[Yourcenar] dialogue avec soi-même, tout en recherchant l'autre» (p.32). A simili conclusioni giunge anche Valeria Emi Mara SGUEGLIA (Chaque homme est contenu tout entier dans chaque fragment de sa vie, pp. 169-186) che, lavorando in parallello su Essais et mémoires e sulla corrispondenza, afferma che la Yourcenar utilizza quest'ultima per conoscersi meglio. Colette GAUDIN (Corres-pondance 1951-1956: affirmation de l'œuvre, double de l'écriture, pp.49-62), ribadendo il concetto del legame lettera-opera, afferma che la lettera ha, per la Yourcenar, lo scopo di fondare e difendere il testo. Bérengère DEPREZ 
(Ce qu'il importait précisément à l'auteur de dire. La correspondance comme paratexte: une stratégie de plus, pp. 33-48) e Marie-Ange JOURDAN-GUEYER (Le «Je» épistolaire de Marguerite Yourcenar, pp.63-78) sostengono la centralità e l'autorevolezza dell'autrice nella sua corrispondenza e Deprez sottolinea come la Yourcenar sfrutti la lettera come mezzo per commentare i propri testi.

Un nutrito gruppo di interventi analizza le relazioni tra l'autrice e i suoi destinatari; in particolare: Daniel LEUWERS (Marguerite Yourcenar et son art d'écrire aux éditeurs, pp. 79-84) e Françoise BONALI FIQUET ( $\mathrm{Du}$ texte à la scène. La Correspondance de Marguerite Yourcenar avec Luca Coppola et Marina Spreafico, pp. 187-202), che mette in luce come l'autrice sia molto benevola nei confronti di coloro che rispettano la sua opera. Maurice DELCROIX, invece, (Lettres (peu) catholiques, pp. 117-130) sottolinea come, nelle lettere di argomento religioso o indirizzate a religiosi, l'autrice sia molto attenta alla sensibilità dei destinatari.

4 I restanti interventi sono abbastanza eterogenei. Anne COUDREUSE (Le Refus du pathos dans la correspondance de Marguerite Yourcenar, pp. 93-108) individua i motivi per cui non c'è, apparentemente, sentimento nelle lettere della Yourcenar. Tre studiosi riflettono, invece, sul rapporto dell'autrice, esiliata volontariamente in America, con l'Europa: André MAINDRON (Sur la culture littéraire de Yourcenar, pp. 131-142), André-Alain MORELLO, (Un amour transatlantique? L'Europe dans les lettres de Marguerite Yourcenar, pp. 143-156) e Patrick HUBNER (L'Arcadie américaine de Marguerite Yourcenar, pp.157-168). Francesca MELZI D'ERIL (À la recherche d'un visage: Antinoüs dans la correspondance de Marguerite Yourcenar, pp. 203-214) e André UGHETTO ("Mémoires d'Hadrien" dans la correspondance de Marguerite Yourcenar, pp.243-256) puntano l'attenzione sulle lettere concernenti i Mémoires, mentre Giorgetto GIORGI ("Denier du rêve" à la lumière de la correspondance de Marguerite Yourcenar, pp. 215-226) sostiene che il progetto di una suite di Denier du rêve, ipotizzata da Marguerite Yourcenar in una lettera a Lidia Storoni Mazzolani, sia stato sostituito dalla scrittura di Labyrinthe du monde. Alexandre TERNEUIL (De l'illustration de soi-même. "L'Album de Fernande" de "Souvenirs pieux" dans la correspondance de Marguerite Yourcenar, pp.227-242) analizza un gruppo di lettere inedite della Yourcenar che chiariscono, tra l'altro, il rapporto tra testo e fotografia secondo l'autrice. Infine, Manuela LEDESMA (Yourcenar, Lilar, Beauvoir et le statut de l'intellectuelle, pp. 257-272), partendo dal carteggio tra Yourcenar e Lilar, si sofferma ad esaminare il ruolo della donna così com'è inteso da queste tre grandi scrittrici e, in appendice, regala agli appassionati una lettera inedita indirizzatale dalla stessa Yourcenar. 